\title{
ON THE SMOOTHNESS OF LEVI-FOLIATIONS
}

\author{
D.E. BARRETT AND J.E. FORNAESS
}

\begin{abstract}
We study the regularity of the induced foliation of a Levi-flat hypersurface in $\mathbb{C}^{n}$, showing that the foliation is as many times continuously differentiable as the hypersurface itself. The key step in the proof given here is the construction of a certain family of approximate plurisubharmonic defining functions for the hypersurface in question.
\end{abstract}

\section{Introduction}

Let $S$ be a real hypersurface in $C^{n}$ of class $C^{k}(k \geq 2)$ with vanishing Leviform. The maximal complex subspace TSกJTS is an integrable distribution of class $C^{k-1}$ and codimension one on $S$, so the Frobenius theorem [5] guarantees that $S$ admits a (unique) foliation of class $C^{k-1}$ by complex hypersurfaces. The goal of this paper is to establish the following result.

Theorem. The induced foliation of a $C^{k}$ Levi-flat hypersurface is actually of class $C^{k}$.

For $k=1$ the Levi-form is not defined, but our proof will nevertheless show that if a real $C^{1}$ hypersurface admits a continuous foliation by complex hypersurfaces then that foliation is of class $C^{1}$. In fact, the existence of the Levi-foliation in the $C^{2}$ case was proved by Shcherbina for $n=2[6]$ and by Airepetian for general $n[1]$; moreover, Airepetian's paper also establishes the $C^{1}$-smoothness of the foliation. It appears that his techrique (which uses both the Bishop disc method and the Frobenius theorem) could also be used to prove our result.

Our proof is based on the construction of a family of approximate pluriharmonic defining functions for $S$. (See the Proposition below). The existence of such functions is also useful in the study of holonomy of Levi-foliations (see $[2])$.

Acknowledgements. The authors wish to thank Franc Forstneric for assistance with translation and Eric Bedford for some helpful remarks. This research was supported in part by an Alfred P. Sloan Foundation Fellowship to the first author and by a NSF Grant to the second author. 


\section{Proof of Theorem}

We work locally near some point in $S$, which we may take to be the origin, and we choose coordinates $z=\left(z^{i}, z_{n}\right), z_{n}=x_{n}+i y_{n}$ so that $T_{0} S=\left\{\left(z^{\prime}, z_{n}\right)\right.$ : $\left.y_{n}=0\right\}$. Then we may write $y_{n}=r\left(z^{\prime}, x_{n}\right)$ on $S$, where $r$ is of class $C^{k}$. Let $\psi\left(t, z^{\prime}\right)$ by defined for $\left(t, z^{\prime}\right)$ near $\left(0,0^{\prime}\right)$ by the condition that $\Psi\left(t, z^{\prime}\right)=$ $=\left(z^{t}, \psi\left(t, z^{\prime}\right)\right)$ lies on the leaf $L_{t}$ passing through $\left(0^{\prime}, t+i r\left(0^{t}, t\right)\right)$. Then $\psi$ is of class $C^{k-1}$ in $\left(t, z^{\prime}\right)$ and holomorphic in $z^{\prime}$. Our goal is to show that $\psi$ is actually of class $C^{k}$ in $\left(t, z^{\prime}\right)$; it is easy to see that all partial derivatives involving fewer than $k$ differentiations with respect to $t$ exist and are continuous, so it will suffice to establish the existence and continuity of $(\partial / \partial t)^{k} \psi$.

The major step in proving the Theorem is the approximation of $S$ along cach leaf $L_{t}$ by the zero set of a pluriharmonic function, as specified in the following Proposition.

Proposition. There is a function $h(t, z)$ defined in a neighborhood $U$ of $(0,0)$ sach that the following conditions hold for $(t, z) \in U$;

(i) $h$ is continuous in $(t, z)$ and holomorphic in $z$,

(ii) $h(t, z)=0$ for $z \in L_{t}$,

(iii) $\partial h / \partial z_{n} \neq 0$, and

(iv) $\operatorname{Im} h=o\left(|h|^{k}\right)$ for $z \in S$ as $z \rightarrow L_{t}$, uniformly in $(t, z)$.

\section{Proof of Proposition:}

We will construct a sequence of functions $h_{0}, h_{1}, \ldots, h_{k}=h$ defined in a neighborhood $U_{j}$ of $(0,0)$ such that the following conditions hold for $(t, z) \in U_{3}$ :

$(i)_{j} h_{j}$ is continuous in $(t, z)$ and holomorphic in $z$,

$(i i)_{j} h_{j}(t, z)=0$ for $z \in L_{t}$,

$(i i i)_{j} \partial h_{j} / \partial z_{n} \neq 0$, and

(iv) $)_{j} \operatorname{Im} h_{j}=o\left(\left|h_{j}\right|^{j}\right)$ for $z \in S$ as $z \rightarrow L_{i}$, uniformly in $(t, z)$.

We may take $h_{0}(t, z)=z_{n}-\psi\left(t, z^{\prime}\right)$.

Let $T=\left(\partial / \partial x_{n}\right)+\left(\partial r / \partial x_{n}\right)\left(\partial / \partial y_{n}\right) ; T$ is a vector field of class $C^{k-1}$ tangent, to $S$ and transverse to the Levi-foliation near 0 . Let

$$
\begin{aligned}
\Theta_{1}\left(t, z^{\prime}\right) & =\arg \left(T h_{0}\right)\left(\Psi\left(t, z^{\prime}\right)\right) \\
& =\arg \left(1+i\left(\partial r / \partial x_{n}\right)\left(\Psi\left(t, z^{\prime}\right)\right) .\right.
\end{aligned}
$$

Then $\Theta_{1}$ is continuous in $\left(t, z^{\prime}\right)$, and the function $\operatorname{Im} e^{-i \Theta_{1}\left(t, z^{\prime}\right)} h_{0}(t, z)$ vanishes along $L_{t}$ as does its derivative with respect to the vector field $T$. Thus

$$
\operatorname{Im} e^{-i \Theta_{1}\left(t, z^{\prime}\right)} h_{0}(t, z)=o\left(\left|h_{0}(t, z)\right|\right)
$$

for $z \in S$ as $z \rightarrow L_{t}$, uniformly on a neighborhood of $(0,0)$. We have $\Theta_{1}\left(0,0^{\prime}\right)=$ $=0$ so that working on a smaller neighborhood we may assume that $\left|\Theta_{1}\right|<$ $<\pi / 4$. 
Claim. $\Theta_{1}\left(t, z^{\prime}\right)$ is pluriharmonic in $z^{\prime}$.

Proof of Claim: It will simplify notation to suppress the parameter $t$ temporarily. Also, it will be useful to perform the change of coordinates

$$
\begin{gathered}
\zeta^{\prime}=z^{\prime} \\
\zeta_{n}=\tilde{x}_{n}+i \tilde{y}_{n}=h_{0}(z) .
\end{gathered}
$$

In the $\zeta$-coordinates $S$ is defined by an equation of the form

$$
\tilde{y}_{n}=\tilde{r}\left(\zeta^{\prime}, \tilde{x}_{n}\right)=\tan \Theta_{1}\left(\zeta^{\prime}\right) \cdot \tilde{x}_{n}+o\left(\left|\tilde{x}_{n}\right|\right)
$$

To show that $\Theta_{1}\left(\zeta^{\prime}\right)$ is pluriharmonic it suffices to show that for every complex-linear disc $\Delta$ near $0^{\prime}$ in $C^{n-1}$ and for every $f$ continuous on $\bar{\Delta}$ and holomorphic on $\Delta$ with $\operatorname{Re} f=\Theta_{1}$ on $\partial \Delta$ we have $\operatorname{Re} f\left(\zeta_{0}^{\prime}\right)=\Theta_{1}\left(\zeta_{0}^{\prime}\right)$, where $\zeta_{0}^{\prime}$ is the center of $\Delta$.

Consider the two-parameter family of discs

$$
\Gamma_{\varepsilon, \lambda}\left(\zeta^{\prime}\right)=\left(\zeta^{\prime}, \varepsilon\left\{e^{i f\left(\zeta^{\prime}\right)}+i \lambda\right\}\right), \zeta^{\prime} \in \Delta
$$

We have

$$
\begin{aligned}
\tilde{y}_{n}-\tilde{r}\left(\zeta^{\prime}, \tilde{x}_{n}\right) & =\varepsilon\left\{\lambda+e^{-\operatorname{Im} f\left(\zeta^{\prime}\right)} \cdot \cos \operatorname{Re} f\left(\zeta^{\prime}\right) \cdot\left(\tan \operatorname{Re} f\left(\zeta^{\prime}\right)-\right.\right. \\
& \left.\left.-\tan \Theta_{1}\left(\zeta^{\prime}\right)\right)\right\}+o(|\varepsilon|)
\end{aligned}
$$

for $\zeta=\Gamma_{e, \lambda}\left(\zeta^{\prime}\right), \zeta^{\prime} \in \Delta$.

Suppose that $\Theta_{1}\left(\zeta_{0}^{\prime}\right)>\operatorname{Re} f\left(\zeta_{0}^{\prime}\right)$. Pick $a$ and $b$ so that

$$
0<a<e^{-\operatorname{Im} f\left(\zeta_{0}^{\prime}\right)} \cdot \cos \operatorname{Re} f\left(\zeta_{0}^{\prime}\right) \cdot\left(\tan \Theta_{1}\left(\zeta_{0}^{\prime}\right)-\tan \operatorname{Re} f\left(\zeta_{0}^{\prime}\right)\right)
$$

and

$$
b>2 \max _{\zeta^{\prime} \in \bar{\Delta}} e^{-\operatorname{Im} f\left(\zeta^{\prime}\right)} .
$$

Then for sufficiently small $\varepsilon>0$ we have

$$
\begin{aligned}
& \tilde{y}_{n}<\tilde{r}\left(\zeta^{\prime}, \tilde{x}_{n}\right) \text { when } \zeta=\Gamma_{\varepsilon, a}\left(\zeta_{0}^{\prime}\right), \\
& \tilde{y}_{n}>\tilde{r}\left(\zeta^{\prime}, \tilde{x}_{n}\right) \text { when } \zeta=\Gamma_{\varepsilon, \lambda}\left(\zeta^{\prime}\right), \zeta^{\prime} \in \partial \Delta, a \leq \lambda \leq b, \\
& \tilde{y}_{n}>\tilde{r}\left(\zeta^{\prime}, \tilde{x}_{n}\right) \text { when } \zeta=\Gamma_{\varepsilon, b}\left(\zeta^{\prime}\right), \zeta^{\prime} \in \bar{\Delta} .
\end{aligned}
$$

But this violates the disc theorem $[4$, p. 53$]$, since $S$ is clearly pseudoconvex from both sides.

The case $\Theta_{1}\left(\zeta_{0}^{\prime}\right)>\operatorname{Re} f\left(\zeta_{0}^{\prime}\right)$ is similar.

Now choose $f_{1}\left(t, z^{\prime}\right)$ continuous in $\left(t, z^{\prime}\right)$ and holomorphic in $z^{\prime}$ with $\Theta_{1}=$ $=\operatorname{Re} f_{1}$. Let $h_{1}(t, z)=e^{i f_{1}\left(t, z^{\prime}\right)} h_{0}(t, z)$. Then $h_{1}$ satisfies $(i)_{1},(i i)_{1},(i i i)_{1}$, and $(i v)_{1}$ on a suitable neighborhood $U_{\mathrm{I}}$ of $(0,0)$. 
Let us assume that $h_{j-1}$ has been constructed and proceed to construct $h_{j}$. Let

$$
\Theta_{j}\left(t, z^{\prime}\right)=\left.\frac{\left(T^{j} \operatorname{Im} h_{j-1}\right)(t, z)}{j !\left(\left(T \operatorname{Re} h_{j-1}\right)(t, z)\right)^{j}}\right|_{z=\Psi\left(t, z^{\prime}\right)}
$$

(Condition $(i i i)_{j-1}$ shows that the denominator doesn't vanish at $\left(0,0^{\prime}\right)$ ). Then $\Theta_{j}$ is continuous in $\left(t, z^{\prime}\right)$ (recall that $z$-derivatives of $h_{j-1}$ come for free), and the function

$$
\operatorname{Im} h_{j-1}(t, z)-\Theta_{j}\left(t, z^{\prime}\right) \cdot\left(\operatorname{Re} h_{j-1}(t, z)\right)^{j}
$$

vanishes along $L_{t}$ along with its derivatives of order $\leq j$ with respect to the vector field $T$. Hence

$$
\operatorname{Im} h_{j-1}(t, z)-\Theta_{j}\left(t, z^{\prime}\right) \cdot\left(\operatorname{Re} h_{j-1}(t, z)\right)^{j}=o\left(\left|h_{j-1}(t, z)\right|^{j}\right)
$$

for $z \in S$ as $z \rightarrow L_{t}$, uniformly on a neighborhood of $(0,0)$.

Claim. $\Theta_{j}\left(t, z^{\prime}\right)$ is plurihormonic in $z^{\prime}$.

Proof of Claim: Again we suppress $t$ temporarily and perform a change of coordinates

$$
\begin{gathered}
\zeta^{\prime}=z^{\prime} \\
\zeta_{n}=\tilde{x}_{n}+i \tilde{y}_{n}=h_{j-1}(z)
\end{gathered}
$$

Thus $S$ is defined by an equation of the form

$$
\tilde{y}_{n}=\Theta_{j}\left(\zeta^{\prime}\right) \cdot \tilde{x}_{n}^{j}+o\left(\left|\tilde{x}_{n}\right|^{j}\right)
$$

As before, it suffices to show that for every complex-linear disc $\Delta$ near $0^{\prime}$ in $C^{n-1}$ and for every $f$ continuous on $\bar{\Delta}$ and holomorphic on $\Delta$ with $\operatorname{Re} f=\Theta_{j}$ on $\partial \Delta$ we have $\operatorname{Re} f\left(\zeta_{0}^{\prime}\right)=\Theta_{j}\left(\zeta_{0}^{\prime}\right)$, where $\zeta_{0}^{\prime}$ is the center of $\Delta$.

Again we consider a two-parameter family of dises

$$
\Gamma_{\varepsilon, \lambda}\left(\zeta^{\prime}\right)=\left(\zeta^{t}, \varepsilon+i \varepsilon^{j}\left\{\lambda+f\left(\zeta^{\prime}\right)\right\}\right), \zeta^{i} \in \Delta
$$

We have

$$
\tilde{y}_{n}-\tilde{\tau}\left(\zeta^{\prime}, \ddot{x}_{n}\right)=\epsilon^{j}\left\{\lambda+\operatorname{Re} f\left(\zeta^{\prime}\right)-\Theta_{j}\left(\zeta^{\prime}\right)\right\}+o\left(|\varepsilon|^{j}\right)
$$

for $\zeta=\Gamma_{\varepsilon, \lambda}\left(\zeta^{t}\right), \zeta^{t} \in \Delta$.

Suppose that $\Theta_{j}\left(\zeta_{0}^{\prime}\right)>\operatorname{Re} f\left(\zeta_{0}^{\prime}\right)$. Pick $a$ and $b$ so that

$$
0<a<\Theta_{j}\left(\zeta_{0}^{\prime}\right)-\operatorname{Re} f\left(\zeta_{0}^{\prime}\right)
$$

and

$$
b>2 \max _{\zeta^{\prime} \in \dot{\Delta}}\left\{\Theta_{j}\left(\zeta^{\prime}\right)-\operatorname{Re} f\left(\zeta^{\prime}\right)\right\}
$$


Then for sufficiently small $\varepsilon>0$ we have

$$
\begin{aligned}
& \tilde{y}_{n}<\tilde{r}\left(\zeta^{\prime}, \tilde{x}_{n}\right) \text { when } \zeta=\Gamma_{\varepsilon, a}\left(\zeta_{0}^{\prime}\right), \\
& \tilde{y}_{n}>\tilde{r}\left(\zeta^{\prime}, \tilde{x}_{n}\right) \text { when } \zeta=\Gamma_{\varepsilon, \lambda}\left(\zeta^{\prime}\right), \zeta^{\prime} \in \partial \Delta, a \leq \lambda \leq b, \\
& \tilde{y}_{n}>\tilde{r}\left(\zeta^{\prime}, \tilde{x}_{n}\right) \text { when } \zeta=\Gamma_{\epsilon, b}\left(\zeta^{\prime}\right), \zeta^{\prime} \in \bar{\Delta} .
\end{aligned}
$$

But this violates the disc theorem as before.

The case $\Theta_{1}\left(\zeta_{0}^{\prime}\right)<\operatorname{Re} f\left(\zeta_{0}^{\prime}\right)$ is again similar.

Again choose $f_{j}\left(t, z^{\prime}\right)$ continuous in $\left(t, z^{\prime}\right)$ and holomorphic in $z^{\prime}$ with $\Theta_{j}=$ $=\operatorname{Re} f_{j}$. Let $h_{j}(t, z)=h_{j-1}(t, z)-i f_{j}\left(t, z^{\prime}\right)\left(h_{j-1}(t, z)\right)^{j}$. Then $h_{j}$ satisfies $(i)_{j},(i i)_{j},(i i i)_{j}$, and $(i v)_{j}$ on a suitable neighborhood $U_{j}$ of $(0,0)$.

The proposition is proved, by induction.

\section{Remarks.}

1) In the case $k=\infty$ it need not be the case that $S$ can be approximated to infinite order along a given leaf by the zero set of a pluriharmonic function. For $n=1$, for example, a $C^{\infty}$ curve need not be approximable to infinite order at a given point by a real-analytic curve.

2) For $j \leq k-2$ the claims in the above proof can be proved by a straightforward Levi-form computation.

3) One can avoid explicit mention of pseudoconvexity in the above proof by observing that the winding number of the boundary of a holomorphic disc around a given leaf cannot jump under small perturbations.

4) The functions $h_{j}$ can actually be chosen to be of class $C^{k-j}$ in $(t, z)$.

5) If $\tilde{h}(z)$ is a holomorphic function vanishing on $L_{0}$ with $\operatorname{Im} \tilde{h}(z)=$ $=o\left(|\tilde{h}(z)|^{k}\right)$ on some neighborhood of 0 in $S$ then

$$
\tilde{h}(z)=P(h(0, z))+\beta(z) \cdot(h(0, z))^{k+1},
$$

where $P$ is a polynomial of degree $k$ with real coefficients and $\beta$ is holomorphic. Indeed, we may write

$$
\tilde{h}(z)=\sum_{j=0}^{k} \alpha,\left(z^{\prime}\right) \cdot(h(0, z))^{j}+\beta(z) \cdot(h(0, z))^{k+1},
$$

where $\beta$ and the $\alpha_{j}$ are holomorphic. Thus

$$
\sum_{j=0}^{k}\left(\operatorname{Im} \alpha_{j}\left(z^{\prime}\right)\right)(\operatorname{Re} h(0, z))^{j}=\operatorname{Im} \tilde{h}(z)+o\left(|h(0, z)|^{k}=o\left(|\operatorname{Re} h(0, z)|^{k}\right)\right.
$$

on $S$, forcing $\operatorname{Im} \alpha_{j} \equiv 0$ for $0 \leq j \leq k$, so that each $\alpha_{j}$ is a real constant.

6) If $S$ is a real hypersurface of class $C^{k}$ which is pseudoconvex from one side and which contains a complex hypersurface then the functions $h_{j}$ can be 
constructed for $j<$ some even integer $j_{0}$; the corresponding function $\Theta_{j_{0}}$ will be sub-or superharmonic. (The pluriharmonicity of $\Theta_{1}$ has been used in several papers, for example in [3, p. 290].)

To prove the Theorem we first note that $\operatorname{Re} h$ has constant sign on each leaf so that by Harnack's inequality we have

$$
\operatorname{Re} h\left(t_{0}, \Psi\left(t, z^{i}\right)\right)=0\left(\left|\operatorname{Re} h\left(t_{0}, \Psi\left(t, 0^{\prime}\right)\right)\right|\right)
$$

for $t_{0}, t, z^{\prime}$ close enough to zero. But (iv) implies that $\operatorname{Re} h$ and $h$ are comparable so it follows that

$$
h\left(t_{0}, \Psi\left(t, z^{\prime}\right)\right)=0\left(\left|h\left(t_{0}, \Psi\left(t, 0^{\prime}\right)\right)\right|\right)
$$

and so

$$
\operatorname{Im} h\left(t_{0}, \Psi\left(t, z^{\prime}\right)\right)=o\left(\left|h\left(t_{0}, \Psi\left(t, 0^{\prime}\right)\right)\right|^{\dot{k}}\right) .
$$

Thus from bounds for pluriharmonic conjugates we have

$$
h\left(t_{0}, \Psi\left(t, z^{\prime}\right)\right)-h\left(t_{0}, \Psi\left(t, 0^{\prime}\right)\right)=o\left(\left|h\left(t_{0}, \Psi\left(t, 0^{\prime}\right)\right)\right|^{k}\right)=o\left(\left|t-t_{0}\right|^{k}\right)
$$

after shrinking the domain of $z^{\prime}$.

By (iii) and the inverse function theorem we may write

$$
z_{n}=\Phi\left(t, z^{\prime}, h(t, z)\right),
$$

where $\Phi$ is continuous in $(t, w)$ and holomorphic in $w$. Thus

$$
\psi\left(t, z^{\prime}\right)=\Phi\left(t_{0}, z^{t}, h\left(t_{0}, \Psi\left(t, z^{\prime}\right)\right)\right)=\Phi\left(t_{0}, z^{\prime}, h\left(t_{0}, \Psi\left(t, z^{\prime}\right)\right)\right)+o\left(\left|t-t_{0}\right|^{k}\right) .
$$

Now the main term of this last expression is $C^{k}$ with respect to $t$, so that the following Lemma will establish the existence and continuity of $(\partial / \partial t)^{k} \psi\left(t, z^{\prime}\right)$ by showing that

$$
(\partial / \partial t)^{k} \psi\left(t, z^{\prime}\right)=\left.(\partial / \partial t)^{k} \Phi\left(t_{0}, z^{\prime}, h\left(t_{0}, \psi\left(t, 0^{\prime}\right)\right)\right)\right|_{t_{0}=t} .
$$

Lemma. Let $f$ be a $C^{k-1}$ function on an interval $I \subset \mathbf{R}$. Suppose that there is a function $g$ on $I \times I$ such that

(i) $(\partial / \partial t)^{j} g(s, t)$ exists and is continuous on $I \times I$ for $0 \leq j \leq k$, and (ii) $f(t)=g(s, t)+o\left(|t-s|^{k}\right)$ uniformly on $I \times I$.

Then

$$
f^{(k)}(t)=\left.(\partial / \partial t)^{k} g(t, s)\right|_{s=t} .
$$

Proof of Lemma: It is clear from the hypotheses that

$$
f^{(j)}(t)=\left.(\partial / \partial t)^{j} g(s, t)\right|_{s=t}
$$

for $0 \leq j \leq k-1$. Let $P(s, t)$ denote the $(k-1)^{s t}$ Taylor polynomial for $f$ at $s$, and let

$$
\alpha(t)=\left.(\partial / \partial t)^{k} g(s, t)\right|_{s=t}
$$


Then applying Taylor's theorem to $g$ in (ii) we have

$$
f(t)=P(s, t)+\alpha(t)(t-s)^{k} / k !+o\left(|t-s|^{k}\right)
$$

uniformly on compact subsets of $I \times I$. Let $\nabla_{h}$ denote the difference operator

$$
\nabla_{h} \varphi(t)=\{\varphi(t+h)-\varphi(t)\} / h .
$$

Applying $\left(\nabla_{h}\right) k-1$ to both sides of $\left(^{*}\right)$ and taking $s=t$ we get

$$
\left(\nabla_{k}\right)^{k-1} f(t)=f^{(k-1)}(t)+c_{k} \alpha(t) h+o(h),
$$

uniformly on compact subsets of $I$, where

$$
c_{k}=\left.h^{-1}\left(\nabla_{h}\right)^{k-1}(t-s)^{k}\right|_{s=t}=(k-I)(k !) / 2 .
$$

Thus

$$
\begin{aligned}
f^{(k)}(t) & =\lim _{h \rightarrow 0} \nabla_{h} f^{(k-1)}(t) \\
& =\lim _{h \rightarrow 0}\left(\nabla_{h}\right)^{k} f(t)-c_{k}\{\alpha(t+h)-\alpha(t)\}+o \\
& =\lim _{h \rightarrow 0}\left(\nabla_{h}\right)^{k} f(t) \\
& =\lim _{h \rightarrow 0} \alpha(t)+o(1) \quad\left(\text { by }\left(^{*}\right)\right. \text { again) } \\
& =\alpha(t) .
\end{aligned}
$$

This completes the proof of the Theorem.

\section{References}

1. R.A. AIRAPETian, Extending $C R$ functions from piecewise smooth $C R$ manifolds, Math. Sbornik 134 (1987), 108-118, Russian.

2. D. BARreTt, Complex analytic realization of Reeb's foliation of $S^{3}$, Preprint.

3. K. DIEDERICH AND J. FornaEss, Pseudoconvex domains: an example with non-trivial nebenhülle, Math. Ann. 225 (1977), 275-292.

4. J. FORNAESS AND B. STENSONES, Lectures on Counterexamples in Several Complex Variables, Mathematical Notes 33, Princeton University Press, Princeton (1987).

5. R. NARASimhan, Analysis on Real and Complex Manifolds, North-Holland, Amsterdam (1968).

6. N.V. ShCherbina, Descomposition of a common boundary of two domains of holomorphy into analytic curves, Iszvestía Akad. Nauk. SSSR Ser. Mat. 46 (1982), 1106-1123; Math. USSR Iszvestia 21 (1983), 399-413.

Institut Mittag-Leffer

Auravägen 17

S I82 62 Djursholm, SWEDEN.

Rebut el 19 de Febrer de 1988 